\title{
High-Temperature Scanning Tunnelling Spectroscopy of Transition Metal Oxides
}

\author{
Z. KLuseK ${ }^{a, b, *}$, P.K. DatTA ${ }^{a}$, P. KOWALCZYK ${ }^{b}$, \\ S. Pierzgalski ${ }^{b}$, A. Busiakiewicz ${ }^{b}$, W. Olejniczak ${ }^{b}$ \\ A. BASU ${ }^{c}$ AND A.W. BRINKMAN ${ }^{c}$
}

${ }^{a}$ Advanced Materials Research Institute (AMRI), University of Northumbria Ellison Building, Ellison Place, Newcastle upon Tyne, NE1 8ST, UK

${ }^{b}$ Department of Solid State Physics, University of Lódź Pomorska 149/153, 90-236 Łódź, Poland

${ }^{c}$ Department of Physics, University of Durham

South Road, DH1 3LE Durham, UK

\begin{abstract}
In situ high-temperature scanning tunnelling spectroscopy measurements recorded on the heavily reduced $\mathrm{TiO}_{2}(110)$ surface which contains $\mathrm{Ti}_{2} \mathrm{O}_{3}$ regions showed disappearance of the energy gap accompanied by substantial decrease in amplitude of the band edge states with increasing temperature. It indicates smooth insulator-metal transition caused by bands overlap in $\mathrm{Ti}_{2} \mathrm{O}_{3}$, which takes place at elevated temperatures. In situ high-temperature scanning tunnelling microscopy and spectroscopy were used to study the influence of temperature on the electronic properties of $\mathrm{Ni}_{x} \mathrm{Mn}_{3-x} \mathrm{O}_{3-\delta}(0.4 \leq x \leq 1)$ thin films deposited by rf magnetron sputtering at three different oxygen/argon $(2.5 \%, 10 \%, 15 \%)$ containing ambient. The morphology and distribution of the local density of states of the observed films did not show any difference for the films deposited at different conditions. The distribution of the local density of states was temperature dependent. The changes in the shape of the local density of states observed at $473 \mathrm{~K}$ were reversible with temperature implying that no permanent change of the electronic structure occurred.
\end{abstract}

PACS numbers: 71.30.+h, 73.20.At, 72.80.Ga

*corresponding author; e-mail: zbklusek@mvii.uni.lodz.pl 


\section{Introduction}

Transition metal oxides (TMO's) are good examples of highly correlated electron systems with very strong coupling between structural (lattice), magnetic (spin), and electronic (charge) properties — see Fig. 1 [1]. This strong coupling leads to amazing response of these functional materials to external conditions such as temperature, magnetic and electric fields. It should be mentioned here that even small variation of external conditions could change the ground state of the system completely altering the properties of these materials.

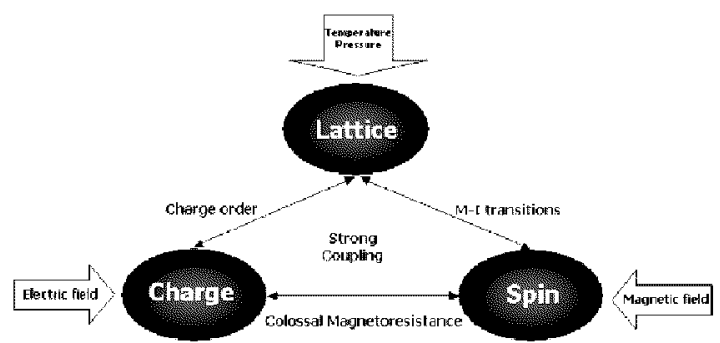

Fig. 1. Schematic presentation of close coupling of spin, charge, and lattice in highly functional materials [1].

One of these transitions is the insulator-metal transition (I-M) marked by a change from the insulating behaviour to the metallic behaviour at certain temperatures [2,3]. Another interesting phenomenon is the negative temperature coefficient of resistance (NTCR) [2, 3]. The mechanisms of the I-M transition and NTCR in transition metal oxides are complex and are still not well understood. The complexity of these phenomena is mainly a result of the many-electron-phonon interactions in oxides and assumptions about the possible shape of the distribution of the local density of states (LDOS) around the Fermi level. Although much effort has been devoted into low temperature studies of the local electronic structure of these materials using scanning tunnelling spectroscopy (STS), the high temperature $(T>300 \mathrm{~K})$ region has remained unresearched.

We show results of our in situ high-temperature scanning tunnelling spectroscopy (HT-STS) studies on heavily reduced $\mathrm{TiO}_{2}(110)$ surface. In stoichiometric rutile $\mathrm{TiO}_{2}$ crystal the metal atom is in $3 d^{0}\left(\mathrm{Ti}^{4+}\right)$ electronic configuration, however the surface can be reduced by ion sputtering or heat treatment to create $\mathrm{Ti}_{2} \mathrm{O}_{3}$ regions in which metal atom is in $3 d^{1}\left(\mathrm{Ti}^{3+}\right)$ configuration. We show that below $T_{c} \approx 450 \mathrm{~K}, \mathrm{Ti}_{2} \mathrm{O}_{3}$ indicates the presence of a small energy gap between the occupied $a_{1 \mathrm{~g}}$ band and the unoccupied $e_{\mathrm{g}}^{\pi}+e_{\mathrm{g}}^{\pi *}$ bands. Above $T_{\mathrm{c}}$ the disappearance of the energy gap accompanied by substantial decrease in amplitude of the band edge states $\left(a_{1 \mathrm{~g}}, e_{\mathrm{g}}^{\pi}+e_{\mathrm{g}}^{\pi *}\right)$ occurs. It indicates smooth insulator-metal transition, which takes place on the surface. 
Finally, we show in situ HT-STS studies $(293 \mathrm{~K}<T<473 \mathrm{~K})$ of thin films of nickel manganese oxide $\left(\mathrm{Ni}_{x} \mathrm{Mn}_{3-x} \mathrm{O}_{4+\delta}(0<x \leq 1)\right)$ deposited on $\mathrm{Si}(100)$ substrates that constitute a family of cubic spinel structured materials, which exhibit NTCR phenomena. In our studies distribution of the local density of states as function of temperature $\operatorname{LDOS}(x, y, E, 300 \mathrm{~K}<T<473 \mathrm{~K})$ was found to be parabolic with energy. This result is consistent with the modified variable range-hopping model for conductivity developed by Shklovskii and Efros [4]. It would appear to exclude the Mott variable range hopping in this material. It is emphasized that the spectra were recorded in the temperature range where this material is most commonly used (thermistors, infrared detecting bolometers).

\section{Experimental}

The STM/STS/CITS experiments were performed at elevated temperatures with a commercial VT-STM/AFM system in UHV condition (Omicron GmbH, Germany) equipped with LEED/Auger spectrometer (SPECTALEED) and a sputtering gun (ISE 5). The tips used were prepared by mechanical cutting from the $90 \% \mathrm{Pt}-10 \%$ Ir alloy wires (Goodfellow). In current imaging tunnelling spectroscopy (CITS) mode, the $I / V$ curves were recorded simultaneously with a constant current image by the interrupted-feedback-loop technique. Based on these measurements the first derivative of the tunnelling current with respect to voltage $(\mathrm{d} I / \mathrm{d} V)$ was calculated. The $\mathrm{d} I / \mathrm{d} V$ spectra were normalised using the method where the differential conductance is divided by the total conductance - $(\mathrm{d} I / \mathrm{d} V) /(I / V)$. The divergence problem in the case of $(\mathrm{d} I / \mathrm{d} V) /(I / V)$ was overcome by applying some amount of broadening $(\Delta V)$ to the $I / V$ values [5].

The $\mathrm{TiO}_{2}(110)$ (Pi-Kem, UK) surface was prepared using repeated cycles of $\mathrm{Ar}^{+}$ion sputtering (typically $30 \mathrm{~min}, 1 \mathrm{keV}$, current $4 \mu \mathrm{A}$ ) and annealing (typically $1050 \mathrm{~K}, 1 \mathrm{~h})$.

The $\mathrm{Ni}_{x} \mathrm{Mn}_{3-x} \mathrm{O}_{4+\delta}$ films on $\mathrm{Si}(100)$ were deposited by rf magnetron sputtering using a $\mathrm{Ni}_{x} \mathrm{Mn}_{3-x} \mathrm{O}_{4+\delta}$ target, made from $\mathrm{NiO}$ and $\mathrm{Mn}_{2} \mathrm{O}_{3}$ precursors following a conventional ceramic powder processing technique [6].

\section{Results and discussion}

\subsection{I-M transition on heavily reduced $\mathrm{TiO}_{2}(110)$ surface}

In generally accepted models the rutile $\mathrm{TiO}_{2}$ is regarded as a large band-gap ( $3 \mathrm{eV}$ ) binary oxide where the valence band $\mathrm{VB}$ can be decomposed into three parts (see Fig. 2): (1V) the $\sigma$ bonding of $\mathrm{O} p_{\sigma}$ and Ti $e_{\mathrm{g}}$ states in the lower energy region; $(2 \mathrm{~V})$ the $\pi$ bonding of the $\mathrm{O} p_{\pi}$ and $\mathrm{Ti} e_{\mathrm{g}}$ states in the middle energy region; $(3 \mathrm{~V})$ the $\mathrm{O} p_{\pi}$ states in the higher energy region $[7,8]$. The resulting band width of the $\mathrm{VB}$ is $5.7 \mathrm{eV}$. The conductance band $\mathrm{CB}$ can be decomposed into two parts: (1C) the bottom of the lower CB consisting of the Ti $d_{x y}$ orbital contribution 
to the metal-metal interactions due to the $\sigma$ bonding of the $\mathrm{Ti} t_{2 \mathrm{~g}}-\mathrm{Ti} t_{2 \mathrm{~g}}$ states, while at the top of the lower $\mathrm{CB}$ the rest of the $\mathrm{Ti} t_{2 \mathrm{~g}}$ states are antibonding with the $\mathrm{O} p_{\pi}$ states; (2C) the upper $\mathrm{CB}$ consists of the $\sigma$ antibonding between the $\mathrm{O} p_{\pi}$ and $\mathrm{Ti} e_{\mathrm{g}}$ states $[7,8]$. The lower group of states (1C) has a band width of $2.9 \mathrm{eV}$, while the upper group has a width of $(2 \mathrm{C}) 3.3 \mathrm{eV}$.

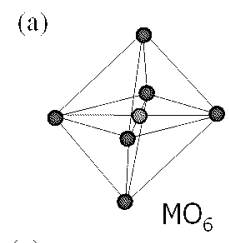

(c)

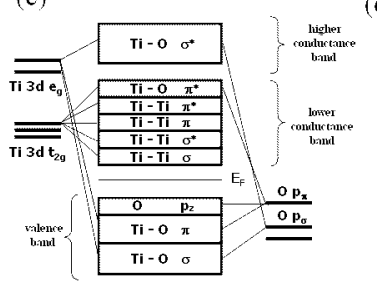

(b)

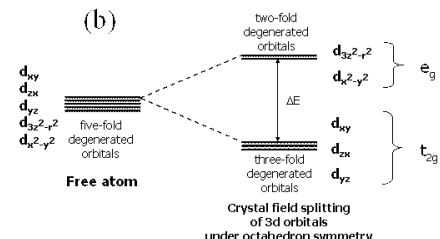

(d)

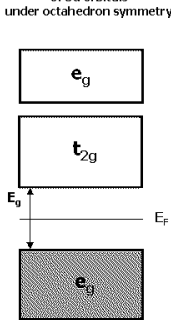

Fig. 2. (a) The basic structural unit of $\mathrm{TiO}_{2}$ (octahedron - $\mathrm{MO}_{6}$ ). (b) Energy level splitting of the Ti 3- $d$ orbitals in the octahedral positions. (c) Bands energy diagram for rutile $\mathrm{TiO}_{2}$. (d) Simple form of band energy diagram for rutile $\mathrm{TiO}_{2}$.

In stoichiometric $\mathrm{TiO}_{2}$ crystals the metal atom is in the $3 d^{0}\left(\mathrm{Ti}^{4+}\right)$ electronic configuration, however the surface can be reduced by ion sputtering or heat treatment to create doubly ionised donor-type oxygen vacancy defects $\left(\mathrm{TiO}_{2-x}\right)$. As a result $\mathrm{TiO}_{2-x}$ can be treated as $n$-type semiconductor with electrons as majority charge carriers. Typical STM images of lightly reduced $\mathrm{TiO}_{2}(110)$ surface with well visible $(1 \times 1)$ superstructure are shown in Fig. 3 and Fig. 4 . The $(1 \times 1)$ superstructure contains two types of oxygen atoms, i.e. atoms at the bridging and in-plane positions, and two types of titanium atoms which are 5 - and 6-fold coordinated. Oxygen atoms bridging and covering the 6 -fold coordinated Ti atoms form ridges along the [001] axis. The 5 -fold coordinated $\mathrm{Ti}$ atoms are exposed to the surface. Thus the $(1 \times 1)$ superstructure consists of alternative rows of the exposed $\mathrm{Ti}$ atoms and the bridging $\mathrm{O}$ ridges. The $\mathrm{O}$ ridges and $\mathrm{Ti}$ rows are aligned with a $0.65 \mathrm{~nm}$ separation, respectively - see Fig. 5. The STM image showing $(1 \times 1)$ superstructure of $\mathrm{TiO}_{2}(110)$ surface exhibiting bright atomic rows parallel to the [001] direction and separated by about $0.7 \mathrm{~nm}$ was recorded at a positive sample bias of $1 \mathrm{~V}$ ( $1 \mathrm{eV}$ above the Fermi level) i.e. the lower conductance band of the $\mathrm{TiO}_{2}$ was probed. From band electronic structure of the $\mathrm{TiO}_{2}$ (see Fig. 2) it is clear that the lower conductance band consists of $\mathrm{Ti} d_{x y}$ orbitals contributing to the metal-metal interactions due to the $\sigma$ bonding of the $\mathrm{Ti} t_{2 \mathrm{~g}}-\mathrm{Ti} t_{2 \mathrm{~g}}$ states. These states are localized on the 5 -fold coordinated Ti atoms exposed to the surface. As 


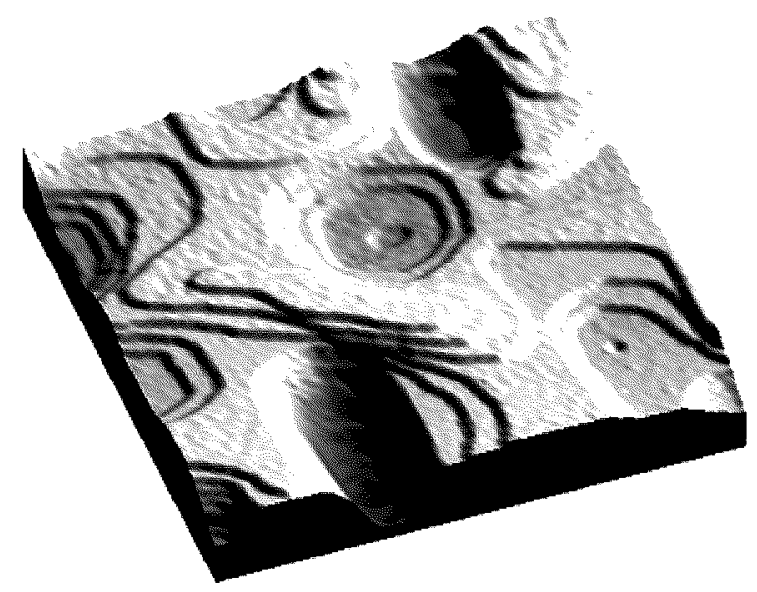

Fig. 3. $30 \mathrm{~nm}^{2}$ STM image of lightly reduced $\mathrm{TiO}_{2}(110)$ surface.

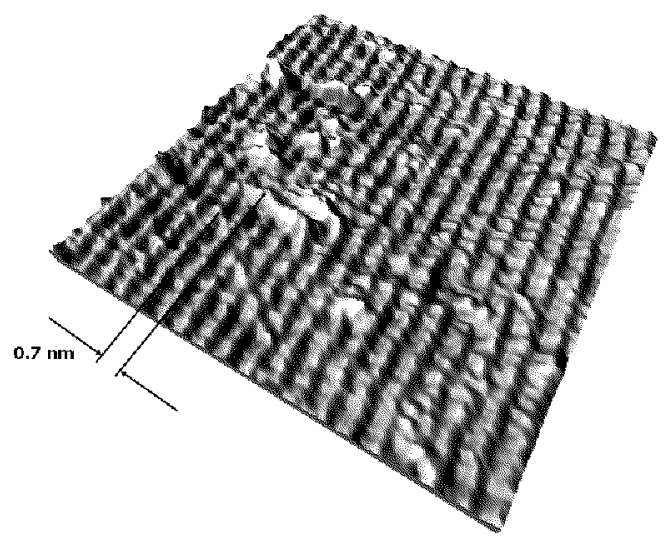

Fig. 4. $15 \mathrm{~nm}^{2}$ STM image of $\mathrm{TiO}_{2}(110)$ surface showing $(1 \times 1)$ reconstruction.

a result $\mathrm{Ti}$ atoms were imaged by STM as bright rows, though the Ti atoms are located $0.11 \mathrm{~nm}$ below the bridging oxygen atoms. It confirms the well-known fact that STM does not show an atomic structure of the surface in crystallographic sense, but rather an atomic structure of the surface, which is strongly affected by the local electronic structure.

Spectroscopic investigations (photoemission spectroscopy (PES), resonant photoemission spectroscopy (ResPES), X-ray excited photoelectron diffraction (XPED), inverse photoemission spectroscopy (IPES)) on reduced $\mathrm{TiO}_{2}(110)$ surface $[9,10]$ have shown the presence of defect states located in the band gap with binding energy below the bulk conduction band edge in $\mathrm{TiO}_{2}$. The $d$ defect state (herewith we use Heise and Courths [10] notation) starts to appear at low density of oxygen vacancies, i.e. for $\mathrm{TiO}_{2-\delta}$ where $\delta \ll 1$. At higher density of vacancies, 


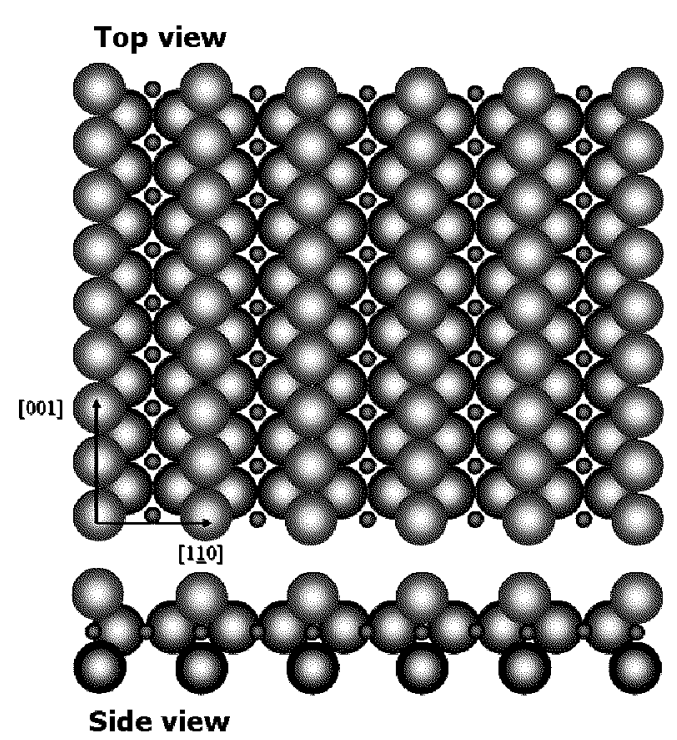

Fig. 5. Atomistic model of the $(1 \times 1)$ superstructure on $\mathrm{TiO}_{2}(110)$ surface.

i.e. for $\mathrm{TiO}_{2-x}$ where $x \gg \delta$, the $D$ defect state can be observed. The presence of defect states is accompanied by a shift of the Fermi level towards higher energy.

In Fig. 6 we show the tunnelling normalised conductance maps measured along three different lines on the $\mathrm{TiO}_{2}(110)$ surface. Figure 7 shows $(\mathrm{d} I / \mathrm{d} V) /(I / V)$ curves taken from the vertical cross-sections in Fig. 6, i.e. for positions marked by $\# 1-\# 2, \# 3-\# 4$, and \#5 lines. All the $(\mathrm{d} I / \mathrm{d} V) /(I / V)$ curves have been shifted in the vertical scale for the sake of clarity. Figure 6 a shows that the $d$ defect state is located in the energetic gap about $1.1 \mathrm{eV}$ below the Fermi level and the energy of the state does not depend strongly on the spatial position. It is also seen that the $d$ state suddenly disappears at $20 \mathrm{~nm}$ from the beginning of the coordinate system. It means that the $d$ state is not uniformly spread on the whole $\mathrm{TiO}_{2}(110)-(1 \times 1)$ surface but is rather confined to some regions. In Fig. $6 \mathrm{~b}$ apart from the $d$ state a new state at energy about $0.55 \mathrm{eV}$ below the Fermi level is clearly observed. We ascribe this state to the presence of the $D$ state which is typical of more reduced $\mathrm{TiO}_{2}(110)$ surface, i.e. $\mathrm{TiO}_{2-x}$ where $x \gg \delta$ condition is fulfilled. Furthermore, as presented in Fig. $6 \mathrm{c}$, the $d$ and $D$ states can be observed simultaneously at the same spatial position. From all the results presented we conclude that at the occupied part of the spectra of the $\mathrm{TiO}_{2}(110)-(1 \times 1)$ surface two distinct surface states are observed, i.e. the $d$ state at energy of about $1.1 \mathrm{eV}$ and the $D$ state at energy of about $0.6 \mathrm{eV}$. The energies of the $d$ and $D$ states estimated from the tunnelling spectroscopy measurements are in good agreement with the previous results recorded on the reduced $\mathrm{TiO}_{2}(110)$ surface by ultraviolet photoemission spectroscopy (UPS) and ResPES measurements [10, 11]. 

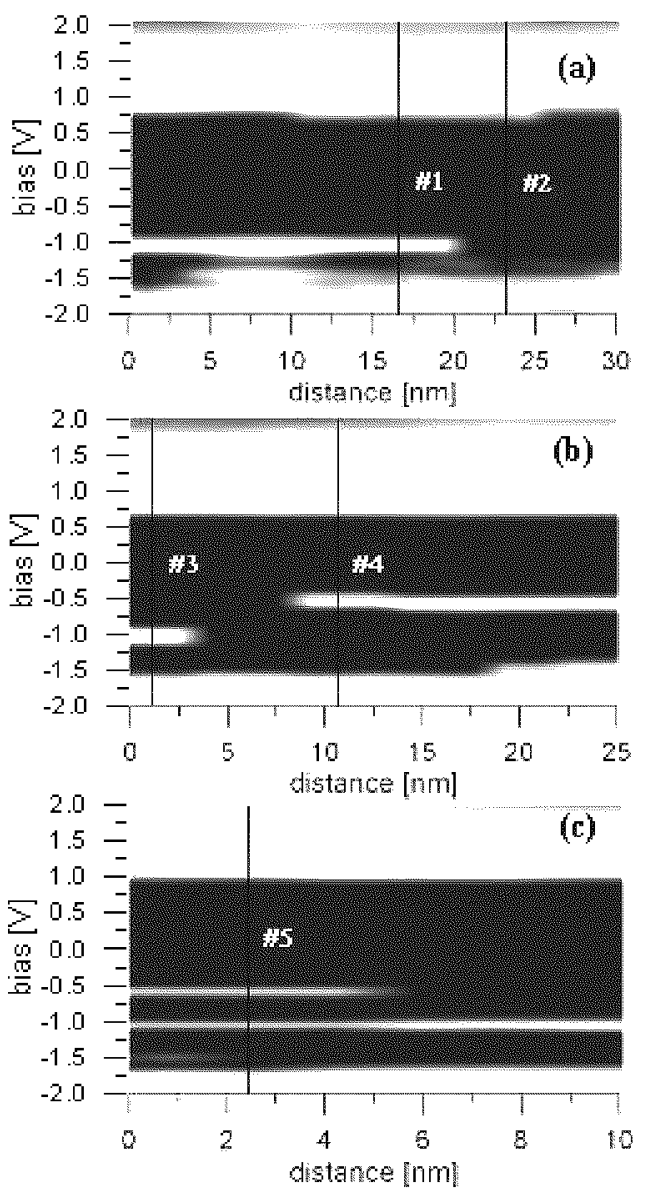

Fig. 6. Normalised tunnelling conductance maps measured as a function of bias voltage and position.

The observed states are the extrinsic surface states caused by the presence of the defects considered in terms of oxygen vacancies and formation of $\mathrm{Ti}^{3+}$ ions on the reduced $\mathrm{TiO}_{2}(110)$ surface. As was shown in [9] the origin of the $d$ state can be attributed to the presence of high density of $\mathrm{Ti}^{3+}$ ions which start to interact with each other and give rise to the formation of the $\mathrm{Ti}^{3+}$ pairs. This process is interpreted in terms of surface phase transition. The $D$ state starts to appear when the density of $\mathrm{Ti}^{3+}$ pairs increases, and small areas of $\mathrm{Ti}_{2} \mathrm{O}_{3}$ on the surface are formed [9].

Furthermore, in tunnelling experiments the $D$ state has the same energy (0.6 eV below the Fermi level) as the energy of the valence band edge $\left(a_{1 \mathrm{~g}}\right)$ in pure $\mathrm{Ti}_{2} \mathrm{O}_{3}[12]$. Thus in our interpretation we assume that the $D$ state and $a_{1 \mathrm{~g}}$ band edge state are the same states, i.e. $D \equiv a_{1 \mathrm{~g}}$. Since $\mathrm{Ti}_{2} \mathrm{O}_{3}$ is treated as a semiconductor with small energy gap between the valence and the conductance 
band edges, then the $D \equiv a_{1 \mathrm{~g}}$ state should be accompanied by the presence of a symmetrically located state at the unoccupied part of the spectra which can be ascribed to the presence of the edge of the conductance band, i.e. $e_{\mathrm{g}}^{\pi}+e_{\mathrm{g}}^{\pi *}$ state. This can be seen from Fig. 7 for the curves \#4 and \#5 where broad structures at energy of about $0.6 \mathrm{eV}$ above the Fermi level are visible.

Further sputtering and annealing of the sample make the surface more reduced (black colour of the sample). It results in loss of oxygen and rearrangements of $\mathrm{Ti}^{3+}$ ions leading to formation $\mathrm{Ti}_{2} \mathrm{O}_{3}$-like $\mathrm{Ti}^{3+}$ pairs $[9,10]$. When the density of pairs increases, areas of $\mathrm{Ti}_{2} \mathrm{O}_{3}$ start to form on the $\mathrm{TiO}_{2}(110)$ surface.

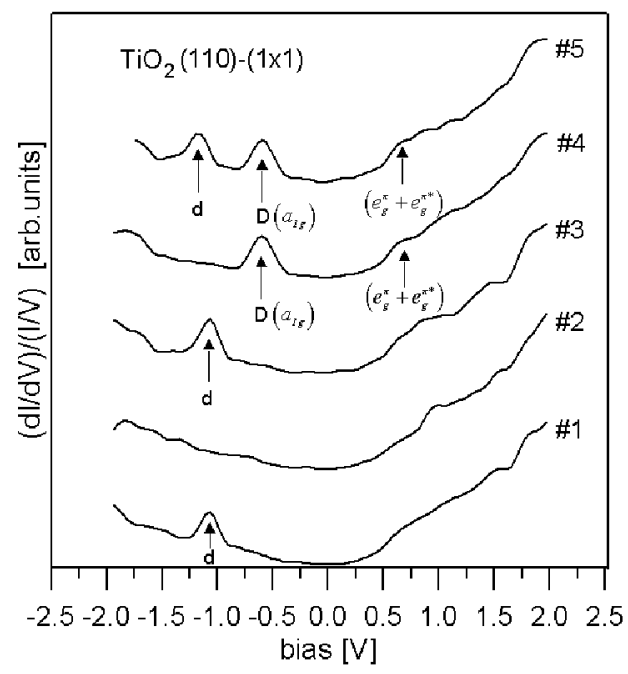

Fig. 7. The $(\mathrm{d} I / \mathrm{d} V) /(I / V)$ curves selected at different spatial positions (\#1-\#5). The \# positions are shown in Fig. 6.

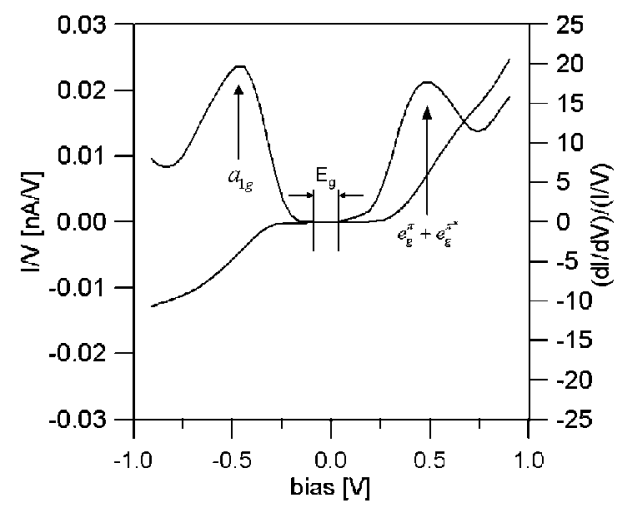

Fig. 8. The $I / V$ curve and its normalized form $(\mathrm{d} I / \mathrm{d} V) /(I / V)$ recorded at low energy range. 
In Fig. 8 we present the $I / V$ curve and its normalized form recorded in a lower energy range where the energy gap, the valence band edge $\left(a_{1 \mathrm{~g}}\right)$, and the conductance band edge $\left(e_{\mathrm{g}}^{\pi}+e_{\mathrm{g}}^{\pi *}\right)$ are well pronounced. This result clearly shows that we are dealing with $\mathrm{Ti}_{2} \mathrm{O}_{3}$ regions on the reduced $\mathrm{TiO}_{2}(110)$ surface. The surface phase transition from $\mathrm{TiO}_{2}$ towards $\mathrm{Ti}_{2} \mathrm{O}_{3}$ is accompanied by a change in the electronic structure as presented in Fig. 9a,b.

Finally, in Fig. 10 we present the $I / V$ curves and their normalized form $(\mathrm{d} I / \mathrm{d} V) /(I / V)$ recorded on heavily reduced $\mathrm{TiO}_{2}(110)$ surface at four tempera-

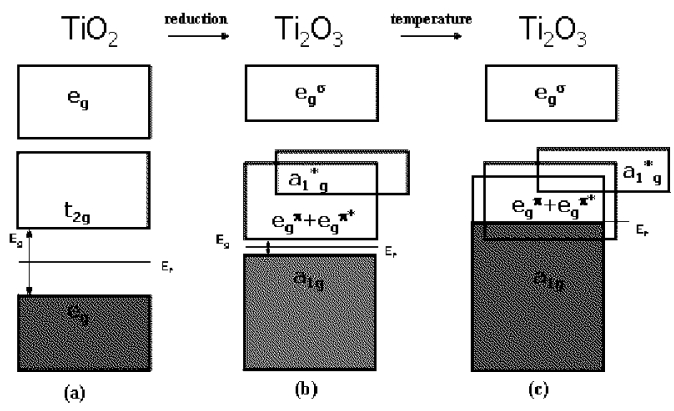

Fig. 9. Band energy diagrams for (a) $\mathrm{TiO}_{2}$, (b) $\mathrm{Ti}_{2} \mathrm{O}_{3}$, and (c) $\mathrm{Ti}_{2} \mathrm{O}_{3}$ above $\mathrm{I}-\mathrm{M}$ phase transition.
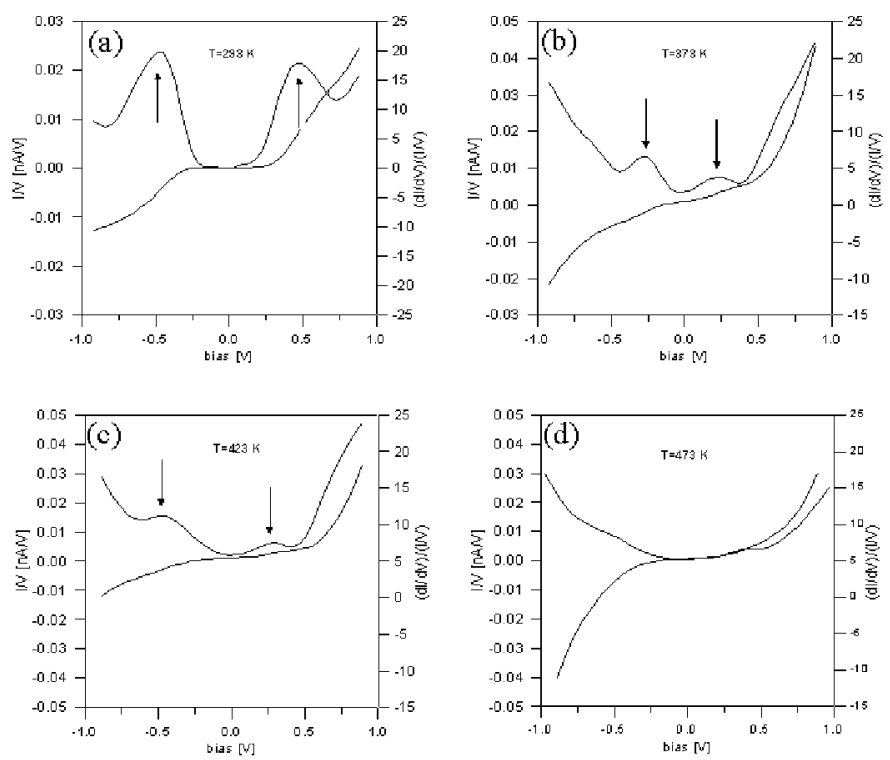

Fig. 10. The $I / V$ curve and its normalized form $(\mathrm{d} I / \mathrm{d} V) /(I / V)$ recorded at (a) $293 \mathrm{~K}$, (b) $393 \mathrm{~K}$, (c) $423 \mathrm{~K}$, and (d) $473 \mathrm{~K}$ on the $\mathrm{TiO}_{2}$ (110) surface. 
tures: $293 \mathrm{~K}, 393 \mathrm{~K}, 423 \mathrm{~K}$, and $473 \mathrm{~K}$. The disappearance of the energy gap accompanied by substantial decrease in amplitude of the band edge states $\left(a_{1 \mathrm{~g}}, e_{\mathrm{g}}^{\pi}+e_{\mathrm{g}}^{\pi *}\right)$ with increasing temperature is clearly seen. It indicates smooth insulator-metal transition, which takes place on the surface. As was observed the disappearance of the energetic gap was completely reversible and it appeared when the temperature was lowered. As was shown in $[13,14]$ the $\mathrm{I}-\mathrm{M}$ transition in $\mathrm{Ti}_{2} \mathrm{O}_{3}$ can be explained by the competition between electron-electron correlation energy and electron-band entropy at elevated temperatures. As a result the $a_{1 \mathrm{~g}}$ and $e_{\mathrm{g}}^{\pi}+e_{\mathrm{g}}^{\pi *}$ bands overlap producing smooth transition with no change in crystal symmetry. A sketch of the $\mathrm{Ti}_{2} \mathrm{O}_{3}$ bands model above the $\mathrm{I}-\mathrm{M}$ transition is shown in Fig. $8 \mathrm{c}$. This clearly explains the disappearance of the energetic gap on high-temperature tunnelling spectroscopy measurements presented in Fig. 10.

$$
\text { 3.2. } H T \text {-STS of } \mathrm{Ni}_{x} \mathrm{Mn}_{3-x} \mathrm{O}_{4+\delta} \text { surface }
$$

Nickel manganese oxides $\mathrm{Ni}_{x} \mathrm{Mn}_{3-x} \mathrm{O}_{4+\delta}(0.4 \leq x \leq 1)$ represent a class of materials, which show negative temperature coefficient of resistance. $\mathrm{Ni}_{x} \mathrm{Mn}_{3-x} \mathrm{O}_{4+\delta}$ crystallises in air to form a cubic spinel phase in the temperature range between $730^{\circ} \mathrm{C}$ and $875^{\circ} \mathrm{C}[15]$. The usefulness of this material in thermistors and infrared detecting bolometers stems from its temperature dependent resistance. Different transport models such as nearest-neighbour hopping [16], variable range hopping [17], and some empirical models [18] have been employed in attempts to describe the electrical conduction processes in NTCR materials. Commonly the observed dependence of the electrical conductivity on the ratio of the trivalent to tetravalent manganese ions in $\mathrm{Ni}_{x} \mathrm{Mn}_{3-x} \mathrm{O}_{4+\delta}$ is believed to be associated with the localised electron hopping from $\mathrm{Mn}^{3+}$ to $\mathrm{Mn}^{4+}$ in the octahedral sites of the lattice [19]. These materials, and nickel manganate in particular, have been studied for their electrical conduction properties, because they are technologically important, although most of these studies have been carried out in bulk ceramic samples. Recently the conduction processes in $\mathrm{Ni}_{x} \mathrm{Mn}_{3-x} \mathrm{O}_{4+\delta}$ have been investigated using thin films [6].

In this paper we report on the structure and the electronic properties of thin films of $\mathrm{Ni}_{x} \mathrm{Mn}_{3-x} \mathrm{O}_{4+\delta}$ obtained using rf magnetron sputtering. Thin films eliminate many of the uncertainties caused by the lack of reproducibility encountered in bulk materials due to porosity and other inhomogeneities. In particular, we examine whether the structure and the electrical properties of the deposited films are influenced by their oxygen content. We have also determined the influence of temperature on the electronic properties of these films at the fundamental level, by carrying out STM studies with in situ sample heating.

Figure 11a shows the scanning tunnelling microscopy results of the films deposited in three oxygen/argon ambients containing respectively $2.5 \%, 10 \%, 15 \%$ oxygen and subsequently annealed at $1073 \mathrm{~K}$ for 1 hour. The results show that the oxygen content did not influence the morphology. The STM results obtained after 

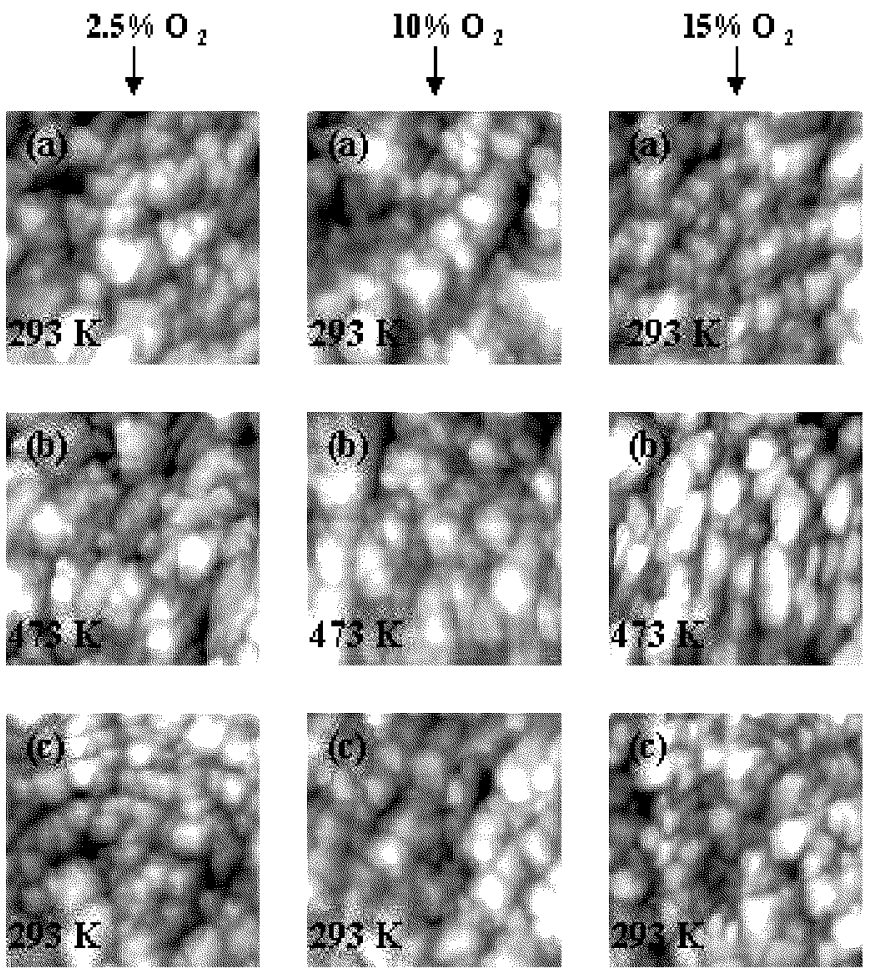

Fig. 11. $600 \mathrm{~nm}^{2}$ STM images of deposited films in various oxygen/argon ambients containing $2.5 \%, 10 \%, 15 \%$ oxygen recorded at different temperatures.

in situ heating at $473 \mathrm{~K}$ and subsequent cooling to $293 \mathrm{~K}$ shown in Fig. $11 \mathrm{~b}$ and $\mathrm{c}$ also demonstrate that the film morphology was not affected by in situ temperature cycling in the range $293 \mathrm{~K} \rightarrow 473 \mathrm{~K} \rightarrow 293 \mathrm{~K}$.

On the same films HT-CITS measurements were performed to investigate the effect of temperature on their electronic structure. The conductance maps of the three types of films at room temperature, at $473 \mathrm{~K}$ and after cooling to room temperature are shown in Fig. $12 \mathrm{a}, \mathrm{b}$ and $\mathrm{c}$. The dark regions correspond to low values of the LDOS. At room temperature (before heating) the characteristics of all the three films looked very similar and the maps suggested that the films behaved like semiconductors with values of the LDOS around the Fermi level that were very close to zero.

From the normalised tunnelling conductance maps it is also possible to extract the individual normalised conductance curves. Figure $13 \mathrm{a}, \mathrm{b}, \mathrm{c}$ show $(\mathrm{d} I / \mathrm{d} V) /(I / V)$ curves taken from vertical cross-sections in the maps in Fig. 12. From these results it is clear that the LDOS had a parabolic shape as previously reported [6] and provide further evidence that this electrical conduction would take place by the variable range hopping model proposed by Shklovskii and Efros [4]. The spectroscopy carried out at $473 \mathrm{~K}$ showed significant differences in com- 

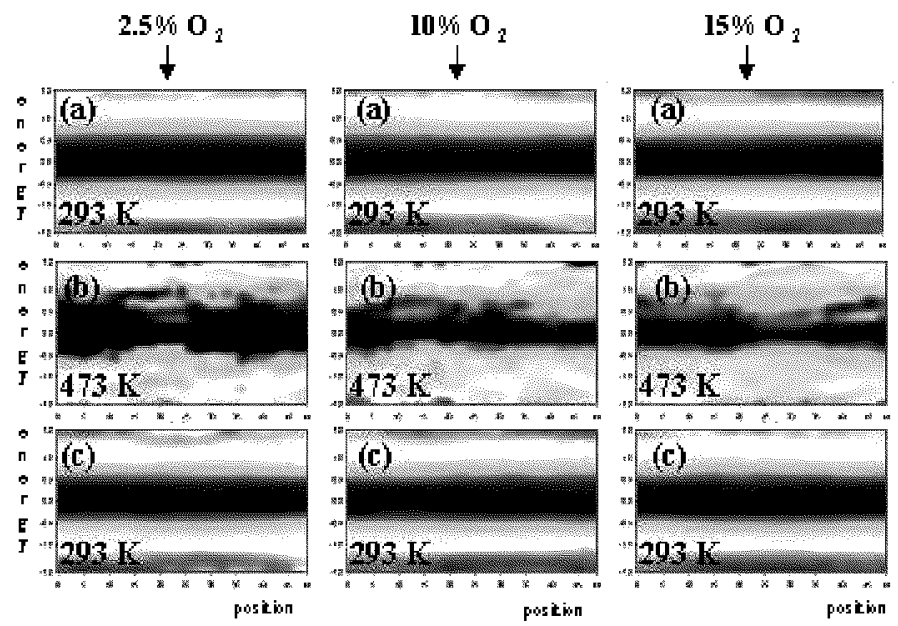

Fig. 12. Conductance maps of deposited films in various oxygen/argon ambients containing $2.5 \%, 10 \%, 15 \%$ oxygen recorded at different temperatures.

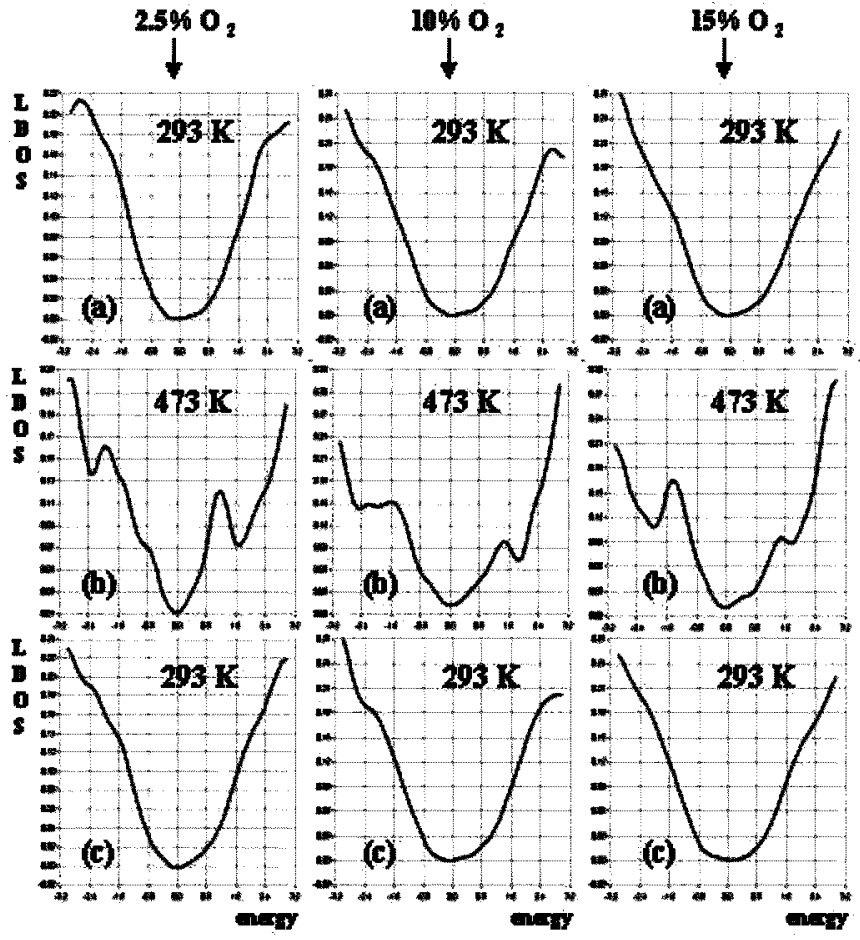

Fig. 13. $(\mathrm{d} I / \mathrm{d} V) /(I / V)$ curves of deposited films in various oxygen/argon ambients containing $2.5 \%, 10 \%, 15 \%$ oxygen recorded at different temperatures.

parison with the results at room temperature. Additional features appeared near the Fermi level, which had not been observed when the spectroscopy was carried 
out at room temperature. It is clear in Fig. 13b (for temperature $473 \mathrm{~K}$ ) that while the basic shape of the LDOS remained parabolic there were additional maxima at energies of $\pm 0.5 \mathrm{eV}$ with respect to the Fermi energy. It has been shown [20] that such features may be associated with local defect states and if so, then it is conceivable that these are the states involved in the electron hopping process that governs transport at elevated temperatures in $\mathrm{Ni}_{x} \mathrm{Mn}_{3-x} \mathrm{O}_{4+\delta}$. The apparent temperature dependence of these features is consistent with the thermal activation of hopping that gives rise to the NTCR effect. The spectroscopic results of the films after cooling to room temperature were similar to the ones recorded before heating and it is apparent that the changes in the shape of the LDOS at $473 \mathrm{~K}$ were reversible with temperature. This ruled out the possible outward diffusion of oxygen from the material because of heating in vacuum, which would alter the stoichiometry and possibly influence the electronic structure. Finally it is important to note that the observed reversibility of electronic properties of the thin films of $\mathrm{Ni}_{x} \mathrm{Mn}_{3-x} \mathrm{O}_{4+\delta}$ is of importance in the design of thermistors.

\section{Conclusions}

Scanning tunnelling microscopy and scanning tunnelling spectroscopy were used to study the electronic structure of the heavily reduced $\mathrm{TiO}_{2}(110)$ surface. At the occupied part of the spectra some states at energies of about $1.1 \mathrm{eV}$ and $0.6 \mathrm{eV}$ below the Fermi level were found. At the unoccupied part of the spectra the presence of a surface state at an energy of about $0.6 \mathrm{eV}$ above the Fermi level was observed. Their presence has been ascribed to the appearance of $\mathrm{Ti}_{2} \mathrm{O}_{3}$ regions on the $\mathrm{TiO}_{2}(110)$ surface. High temperature spectroscopy measurements indicated smooth insulator-metal transition caused by bands overlap in $\mathrm{Ti}_{2} \mathrm{O}_{3}$, which takes place at elevated temperatures.

The morphology and distribution of the LDOS of the observed $\mathrm{Ni}_{x} \mathrm{Mn}_{3-x} \mathrm{O}_{4+\delta}$ films did not show any difference for the films deposited in ambients with different amounts of oxygen. The parabolic nature of the distribution of LDOS is consistent with the underlying assumption used for the variable range hopping model proposed by Shklovskii and Efros describing the conduction processes in such types of materials. The appearance of temperature dependent features in the LDOS indicates the presence of localised electronic states that may be important in the conduction mechanisms. The changes in the shape of the LDOS observed at $473 \mathrm{~K}$ were reversible with temperature implying that no permanent change of the electronic structure occurred.

\section{Acknowledgments}

This work was carried out at the Advanced Material Research Institute, University of Northumbria, supported by grants from the European Regional Department Founding and the University Innovation Centre for Nanotechnology. 


\section{References}

[1] E.W. Plumer, A. Ismail, R. Matzdorf, A.V. Melechko, J.P. Pierce, J. Zhang, Surf. Sci. 500, 1 (2002).

[2] N. Tsuda, N. Nasu, A. Yanase, K. Siratori, Electric Conduction in Oxides, Springer Series in Solid-State Sciences 94, Springer-Verlag, Berlin 1991.

[3] M. Imada, A. Fujimori, Y. Tokura, Rev. Mod. Phys. 70, 1039 (1998).

[4] B. Shklovskii, A. Efros, Electronic Properties of Doped Semiconductors, Springer-Verlag, Berlin 1984.

[5] R. Feenstra, Phys. Rev. B 50, 4561 (1994).

[6] A. Basu, A. Brinkmann, Z. Klusek, P.K. Datta, P. Kowalczyk, J. Appl. Phys. 92, 4123 (2002).

[7] K.M. Glassford, J.R. Chelikowsky, Phys. Rev. B 46, 1284 (1992).

[8] P.I. Soratin, K. Schwarz, Inorg. Chem. 31, 567 (1992).

[9] V.E. Henrich, G. Dresselhaus, H.J. Zeiger, Phys. Rev. Lett. 36, 1335 (1976).

[10] R. Heise, R. Courths, Adsorption in Ordered Surfaces of Ionic Solids and Thin Films, in Springer Series in Surface Science, Vol. 33, Eds. E. Umbach, H.J. Freund, Springer-Verlag, Berlin 1993.

[11] Z. Zhang, S. Jeng, V.E. Henrich, Phys. Rev. B 43, 12004 (1991).

[12] K.E. Smith, V.E. Henrich, Phys. Rev. B 38, 5965 (1988).

[13] H. Zeiger, Phys. Rev. B 11, 5132 (1975).

[14] H. Nakatsugawa, E. Iguchi, Phys. Rev. B 56, 12931 (1997).

[15] D.G. Wickham, J. Inorg. Nucl. Chem. 26, 1369 (1964).

[16] J. Topfer, A. Feltz, D. Graf, B. Hackl, L. Raupach, P. Weissbrodt, Phys. Status Solidi A 134, 405 (1992).

[17] N.F. Mott, J. Non-Cryst. Solids 1, 1 (1968).

[18] G. Bosson, F. Gutmann, L. Simmomson, J. Appl. Phys. 21, 1267 (1950).

[19] E.D. Macklen, Thermistors, Electrochemical Pub., Glasgow 1979.

[20] X. de la Broïse, C. Deleru, M. Lannou, B. Grandidier, D. Suévenard, Phys. Rev. $B$ 61, $2138(2000)$. 